\title{
Critérios para Escolha de Distribuições de Probabilidades em Estudos de Eventos Extremos de Precipitação
}

\author{
Marcel Carvalho Abreu ${ }^{1}$, Roberto Avelino Cecílio ${ }^{2}$, Fernando Falco Pruski ${ }^{3}$, \\ Gérson Rodrigues dos Santos ${ }^{4}$, Laura Thebit de Almeida ${ }^{3}$, Sidney Sara Zanetti ${ }^{2}$ \\ ${ }^{I}$ Departamento de Ciências Ambientais, Instituto de Florestas, Universidade Federal Rural do \\ Rio de Janeiro, Seropédica, Rio de Janeiro, Brasil. \\ ${ }^{2}$ Departamento de Ciências Florestais e da Madeira, Centro de Ciências Agrárias e Engenharias, \\ Universidade Federal do Espírito Santo, Jerônimo Monteiro, Espírito Santo, Brasil. \\ ${ }^{3}$ Departamento de Engenharia Agrícola, Centro de Ciências Agrárias, Universidade Federal de \\ Viçosa, Viçosa, Minas Gerais, Brasil. \\ ${ }^{4}$ Departamento de Estatística, Centro de Ciências Exatas e Tecnológicas, Universidade Federal \\ de Viçosa, Viçosa, Minas Gerais, Brasil.
}

Recebido em: 9 de Março de 2018 - Aceito em: 23 de Setembro de 2018

\begin{abstract}
Resumo
Este estudo objetivou estabelecer um critério sobre qual teste de aderência deve ser preferido na escolha de funções de distribuição de probabilidades (fdp). Para tal, foram ajustadas as fdp: Gumbel (GUM), Generalizada de valores extremos (GEV) e Log-normal a 2 parâmetros (LN2), através dos métodos dos momentos, momentos-L e máxima verossimilhança, em séries de precipitação diária máxima anual de 11 estações localizadas na bacia hidrográfica do rio Sapucaí. A aderência dessas fdp aos dados foi feita pelos testes de Kolmogorov-Smirnov (KS), Qui-quadrado $\left(\chi^{2}\right)$, Filliben (Fi) e Anderson-Darling (AD). Verificaram-se quais testes de aderência são mais rigorosos na seleção de distribuições de probabilidade e, ainda, os testes de aderência que convergem os resultados para a escolha da fdp com melhor desempenho na análise de incerteza e/ou nas estatísticas de ajuste. Os testes de aderência mais rigorosos no aceite da aderência da fdp aos dados são os testes de Fi e AD. O teste de Fi é o que mais converge para a escolha da fdp com melhor desempenho na análise de incerteza e nas estatísticas de ajuste, seguido pelo teste de $\chi^{2}$, portanto devem ser preferidos. As fdp GUM e GEV se destacaram em representar os dados de precipitação máxima anual.
\end{abstract}

Palavras-chave: teste de aderência, modelagem probabilística, chuvas intensas, inferência estatística.

\section{Criteria For Choosing Probability Distributions in Studies of Extreme Precipitation Events}

\begin{abstract}
This study aimed to establish a criterion on which good of fit test should be preferred in the choice of probability distribution functions (pdf). For this, the Gumbel (GUM), Generalized Extreme Value (GEV) and 2-parameter Log-Normal (LN2) were fit Moments, L-moments and maximum likelihood methods, in annual maximum daily precipitation series of 11 rain gauges located in the hydrographic basin of the Sapucaí river. The adherence of these fdp to the data was made by the Kolmogorov-Smirnov (KS), Chi-square $\left(\chi^{2}\right)$, Filliben (Fi) and Anderson-Darling (AD) tests. It was verified which good of fit tests are more rigorous in the selection of pdf and also the good of fit test that converge the results to the choice of the best performing fdp in the uncertainty analysis and/or statistics performance. The most stringent good of fit test on the adherence of fdp to the data are the Fi and AD tests. The Fi test, followed by the $\chi^{2}$ test showed best performance in terms of goodness of fit and convergence therefore, they should be preferred to others tests. The GUM and GEV are great pfd in representing the annual maximum precipitation data.
\end{abstract}

Keywords: goodness of fit test, probabilistic modeling, intense rainfall, statistical inference.

Autor de Correspondência: Marcel Carvalho Abreu, marcelc.abreu@gmail.com. 


\section{Introdução}

A aplicação de modelagem probabilística em estudos hidrológicos é extremamente importante no que tange a previsão de eventos extremos associados a um período de retorno ou a determinada frequência de ocorrência, como as vazões mínimas ou máximas (Lopes et al., 2016) ou os extremos de precipitação (Caldeira et al., 2015; Junqueira Júnior, Mello e Alves,2015) pois estas são variáveis aleatórias contínuas. A análise de frequência em hidrologia está associada a aplicações práticas no manejo, planejamento e gestão de recursos hídricos, tais como o controle de vazões de cheia, os projetos de conservação de água e solo, a drenagem urbana, dentre outros (Beskow, Norton e Mello, 2013). A análise de frequência de eventos hidrológicos envolve basicamente os seguintes passos: a determinação de funções densidade de probabilidade (fdp) a serem testadas; a estimativa dos parâmetros dessas distribuições; a escolha da fdp mais adequada através de testes de aderência; e a verificação das incertezas que o modelo proporciona (Zeng et al. 2015).

Existem diversas fdp que podem ser ajustadas às variáveis hidrológicas aleatórias contínuas. Por sua vez, variáveis hidrológicas podem ser representadas por mais de uma fdp (Beskow et al., 2015). Dentre as principais distribuições probabilísticas destacam-se, por seu emprego em estudos hidrológicos, as seguintes fdp: Generalizada de Eventos Extremos (GEV), Gumbel (GUM), Log-normal a 2 parâmetros (LN2), Log-normal a 3 parâmetros, Pearson a 3 parâmetros, Exponencial, Normal, Generalizada Logística, Gamma, Weibull e Log-Pearson a 3 parâmetros (Valverde et al., 2004).

$\mathrm{O}$ ajuste de distribuições de probabilidade ao conjunto de dados hidrológicos envolve a estimativa dos seus parâmetros. Tal ajuste pode ser realizado por diferentes métodos estatísticos, os quais se destacam o Método dos Momentos (MM), o Método dos Momentos-L (ML) e o Método da Máxima Verossimilhança (MV). Todavia, sabe-se que o método utilizado para estimar os parâmetros das distribuições de probabilidade influencia no grau de ajuste à série de dados (Franco et al., 2014; Caldeira et al., 2015). Portanto, é necessário averiguar qual o melhor método para estimar os parâmetros, a fim de evitar estimadores sujeitos a variações amostrais devido à baixa densidade de estações e ao curto período de análise (Valverde et al., 2004).

Após o ajuste das distribuições de probabilidade, geralmente utilizam-se de testes de aderência para dar suporte à tomada de decisão sobre a qualidade do ajuste de uma fdp à série de dados hidrológicos (Marques et al., 2014). Os testes de aderência mais comumente utilizados são o de Kolmogorov-Smirnov (KS), o Qui-quadrado $\left(\chi^{2}\right)$, o de Filliben (Fi) e o de Anderson-Darling (AD) (Ben-Zvi, 2009; Franco et al., 2014; Beskow et al., 2015). Cada teste de aderência apresenta suas características, as quais irão interferir no rigor destes em rejeitar ou não a hipótese nula de aderência do modelo probabilístico à série de dados. $\mathrm{O}$ teste de KS, por exemplo, é extremamente conservador em rejeitar a hipótese nula (Naghettini e Pinto, 2007). Diversos autores classificam o teste de KS como um teste qualitativo, que estabelece apenas a conclusão sobre a adequabilidade da distribuição em representar o conjunto de dados, sem embasamento para comparar o ajuste de diferentes fdp (Beskow et al., 2015; Caldeira et al., 2015). Já o teste de $\chi^{2}$ é considerado quantitativo (Beskow et al., 2015), porém, tem a decisão do teste influenciada pela forma utilizada para determinação do número e amplitude de classes. O teste de Fi, para amostras de tamanho relativamente pequeno (Narsky, 2003), tende em ter o poder reduzido (Naghettini e Pinto, 2007). Em geral, os testes de $\mathrm{KS}, \chi^{2}$ e Fi são deficientes em discernir as diferenças entre as frequências teóricas e empíricas nas caudas superior e inferior das distribuições em análise, exatamente regiões de interesse em estudos hidrológicos. O teste de AD é uma alternativa interessante (Marques et al., 2014) na aplicação associada a eventos hidrológicos extremos, uma vez que sua estatística utiliza a soma de quadrados das diferenças entre o empírico e o teórico com um fator de ponderação, que evita discrepâncias nas extremidades nas caudas (Shin et al., 2012). Porém, o teste de AD tem restrição quanto à estatística de valor crítico, o qual é conhecido apenas para algumas fdp (Naghetini e Pinto, 2007).

Em relação à análise das incertezas presentes nas estimativas de quantis de interesse, uma medida que pode quantificar a variabilidade presente na estimação das características e parâmetros populacionais é o erro padrão da estimativa $\left(\mathrm{S}_{\mathrm{T}}\right)$, inerente à própria distribuição e método de ajuste dos seus parâmetros (Kite, 1987; Naghettini e Pinto, 2007). Porém, a análise de incertezas já está associada à estimativa do quantil de interesse e nem sempre é considerada na escolha da distribuição de probabilidades a representar os dados.

Outra abordagem utilizada para verificar o desempenho e comparar distribuições de probabilidade é a utilização de estatísticas de ajuste (Martins et al., 2014). As estatísticas de ajuste podem indicar a capacidade de um modelo em representar a realidade através da análise da correlação entre variáveis, indicando o quanto as distribuições probabilísticas estão livres de erros ou tendências. Portanto, essas estatísticas de ajuste podem ser utilizadas de forma complementar aos testes de aderência.

Em estudos hidrológicos, diversos trabalhos utilizaram um ou mais testes de aderência como critério de escolha da melhor distribuição de probabilidade (Franco et al., 2014; Pereira et al., 2014; Damé et al., 2016; Lopes et al., 2016). Outros testaram o quão rigorosos é cada teste de aderência em rejeitar ou não à hipótese de aderência da distribuição à série de dados (Beskow et al., 2015; Junqueira Júnior et al., 2015; Zeng et al., 2015). Apesar das conclusões a respeito de testes mais ou menos rigorosos, 
ainda não se tem indícios sobre o melhor critério para a escolha da melhor fdp ou sobre qual teste converge o resultado para a escolha da distribuição que proporciona menor incerteza e melhor ajuste.

Nesse sentido, o objetivo geral deste trabalho foi, a partir do estudo de séries históricas de chuvas máximas, averiguar quais testes de aderência convergem para a escolha de uma fdp que ofereça o menor erro padrão da estimativa dos quantis de interesse (melhor desempenho na análise de incertezas) e melhor desempenho nas estatísticas de ajuste, visando estabelecer um critério para a escolha da distribuição de probabilidades com melhor desempenho. Os objetivos gerais são: averiguar quais os testes de aderência são mais rigorosos na aceitação da hipótese de aderência das fdp ao conjunto de dados observados e averiguar quais as fdp obtiveram melhores desempenhos nos testes de aderência, em representar os dados observados.

\section{Material e Métodos}

As séries históricas utilizadas neste trabalho são das precipitações diárias máximas anuais (Pmax) de 11 estações Fig. 1 e Tabela 1), todas inseridas na bacia hidrográfica do rio Sapucaí Fig. 1), majoritariamente localizada no estado de Minas Gerais, com uma pequena porção no estado de São Paulo (Almeida et al., 2017).

A bacia do rio Sapucaí apresenta as seguintes classificações climáticas de Köppen: Cfb (clima subtropical) e Cwa (clima tropical de altitude) (Alvares et al., 2013), que indicam, respectivamente, ausência de estação seca com temperatura do mês mais quente inferior a $22^{\circ} \mathrm{C}$ e inverno seco com temperatura do mês mais quente superior a $22^{\circ} \mathrm{C}$. Existe variabilidade na própria bacia em termos de temperatura média (entre $16{ }^{\circ} \mathrm{C}$ e $22^{\circ} \mathrm{C}$ ) e em termos de precipitação média anual (entre $1300 \mathrm{~mm}$ a $1900 \mathrm{~mm}$ ). Para o período em análise, as médias dos totais anuais precipitados variaram entre $1350 \mathrm{~mm}$ e $1512 \mathrm{~mm}$ (Tabela 1).

Localizada em uma das regiões mais úmidas e com maiores índices pluviométricos do sudeste brasileiro, a bacia do rio Sapucaí sofre influencia do Sistema de Monção da América do Sul (Reboita et al., 2017), Zona de Convergência do Atlântico Sul (Carvalho, Jones e Liebmann, 2004), frentes frias (Reboita et al., 2015), além da influência orográfica da serra da Mantiqueira (Almeida et al., 2017). Outra importante informação sobre a bacia é que ela apresenta histórico de enchentes (Almeida et al., 2017) e está sujeita a ocorrência de eventos extremos de chuva entre a primavera e o outono devido a atuação do Sistema de Monção da América do Sul (Marengo et al., 2010; Reboita et al., 2017) e da Zona de Convergência do

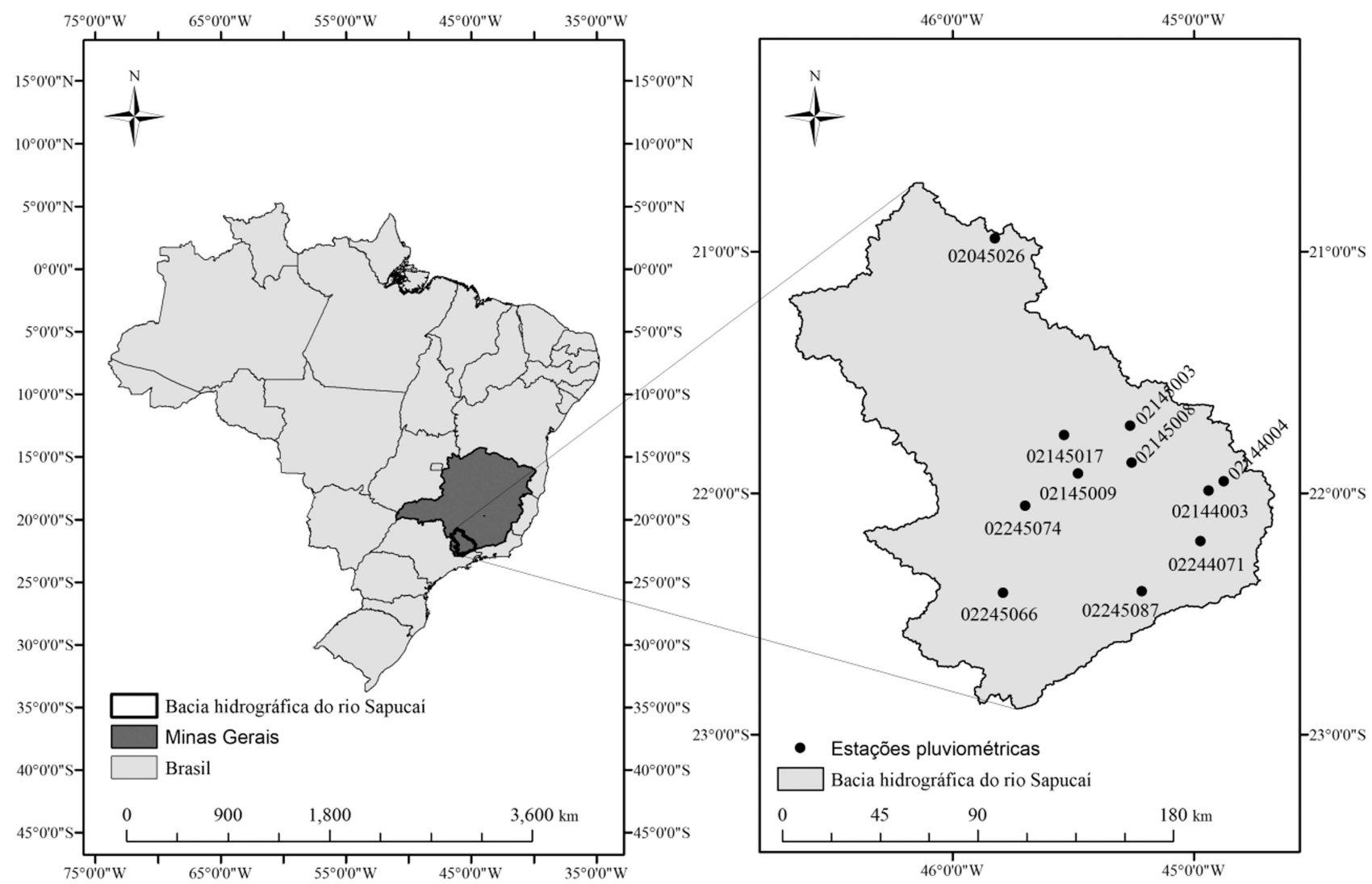

Figura 1 - Localização da bacia hidrográfica do rio Sapucaí e das estações selecionadas para o estudo. 
Tabela 1 - Estações pluviométricas e séries utilizadas neste estudo, porcentagem média de falhas anuais e precipitação média anual para o período analisado.

\begin{tabular}{|c|c|c|c|c|c|c|c|c|}
\hline Código & Nome & Latitude & Longitude & Altitude & Período & $\begin{array}{l}\text { Número de } \\
\text { anos }\end{array}$ & $\begin{array}{l}\text { Falha média } \\
\text { anual }(\%)\end{array}$ & $\begin{array}{l}\text { Precipitação média anual } \\
(\mathrm{mm})\end{array}$ \\
\hline 02144004 & Baependi & $-21^{\circ} 57^{\prime} 04^{\prime \prime}$ & $-44^{\circ} 52^{\prime} 34^{\prime \prime}$ & $880 \mathrm{~m}$ & $1962-1988$ & 27 & 0,00 & 1413,44 \\
\hline 02245087 & Bairro Santa Cruz & $-22^{\circ} 24^{\prime} 25^{\prime \prime}$ & $-45^{\circ} 12^{\prime} 54^{\prime \prime}$ & $1083 \mathrm{~m}$ & $1968-1988$ & 21 & 0,81 & 1511,80 \\
\hline 02144003 & Caxambu & $-21^{\circ} 59^{\prime} 23^{\prime \prime}$ & $-44^{\circ} 56^{\prime} 19^{\prime \prime}$ & $912 \mathrm{~m}$ & $1951-1988$ & 38 & 0,67 & 1465,46 \\
\hline 02245066 & Conceição dos Ouros & $-22^{\circ} 24^{\prime} 51^{\prime \prime}$ & $-45^{\circ} 47^{\prime} 27^{\prime \prime}$ & $850 \mathrm{~m}$ & 1941-1988 & 48 & 0,42 & 1379,97 \\
\hline 02145008 & Fazenda Juca Casemiro & $-21^{\circ} 52^{\prime} 27^{\prime \prime}$ & $-45^{\circ} 15^{\prime} 30^{\prime \prime}$ & $875 \mathrm{~m}$ & 1968-1999 & 32 & 0,00 & 1493,45 \\
\hline 02045026 & Ilicínea & $-20^{\circ} 56^{\prime} 43^{\prime \prime}$ & $-45^{\circ} 49^{\prime} 25^{\prime \prime}$ & $880 \mathrm{~m}$ & $1990-2005$ & 16 & 0,00 & 1349,61 \\
\hline 02145017 & Monsenhor Paulo & $-21^{\circ} 45^{\prime} 37^{\prime \prime}$ & $-45^{\circ} 32^{\prime} 16^{\prime \prime}$ & $810 \mathrm{~m}$ & $1968-2004$ & 37 & 0,33 & 1376,00 \\
\hline 02244071 & Pouso Alto & $-22^{\circ} 11^{\prime} 57^{\prime \prime}$ & $-44^{\circ} 58^{\prime} 24^{\prime \prime}$ & $876 \mathrm{~m}$ & 1967-1999 & 33 & 0,17 & 1504,91 \\
\hline 02145003 & Três Corações & $-21^{\circ} 43^{\prime} 15^{\prime \prime}$ & $-45^{\circ} 15^{\prime} 52^{\prime \prime}$ & $841 \mathrm{~m}$ & 1970-1999 & 30 & 0,04 & 1455,35 \\
\hline 02245074 & $\begin{array}{l}\text { UHE Mar. Mascarenhas de } \\
\text { Moraes }\end{array}$ & $-22^{\circ} 03^{\prime} 12^{\prime \prime}$ & $-45^{\circ} 41^{\prime} 57^{\prime \prime}$ & $770 \mathrm{~m}$ & 1976-1999 & 24 & 0,00 & 1476,15 \\
\hline 02145009 & Usina do Chicão & $-21^{\circ} 55^{\prime} 09^{\prime \prime}$ & $-45^{\circ} 28^{\prime} 44^{\prime \prime}$ & $892 \mathrm{~m}$ & 1962-1999 & 38 & 1,04 & 1491,95 \\
\hline
\end{tabular}

Atlântico Sul (Garcia e Kayano, 2009; Reboita et al., 2017).

Os dados foram obtidos no banco de dados hidrometeorológicos da Agência Nacional das águas (ANA), disponíveis no portal Hidroweb - Sistema de Informações Hidrológicas (http://hidroweb.ana.gov.br/). Como critérios para a utilização das séries de dados considerou-se apenas o uso de dados consistidos, com no mínimo 15 anos, não sendo admitidos anos com porcentagem de falhas anuais superiores a 10\%. Para evitar o descarte de informações, não foi estabelecido um período de análise de dados igual entre as estações. Também, a utilização de séries de diferentes tamanhos é desejável neste tipo de estudo, uma vez que as conclusões não ficariam limitadas a extensão da série. A porcentagem média de falhas anual para cada estação analisada encontra-se na Tabela 1.

Às séries de dados de Pmax foram ajustadas as seguintes distribuições de probabilidade: Generalizada de Valores Extremos (GEV), Gumbel (GUM) e Log-normal a 2 parâmetros (LN2). A estimação dos parâmetros das distribuições foram realizadas pelo método dos momentos (MM), momentos-L (ML) e máxima verossimilhança (MV) (exceto para a distribuição LN2 a qual não se aplicou o método ML) (Junqueira Júnior et al., 2007; Franco et al., 2014; Marques et al., 2014; Beskow et al., 2015; Caldeira et al., 2015).

Posteriormente, aplicaram-se os testes de aderência de Kolmogorov-Smirnov (KS), de Qui-quadrado $\left(\chi^{2}\right)$, de Filliben (Fi) e de Anderson-Darling (AD) para verificar a aderência de cada distribuição, para cada método de estimação dos parâmetros testado. $\mathrm{O}$ nível de significância utilizado em todos os testes foi de $5 \%$.

$\mathrm{O}$ teste de KS testa a hipótese de que as frequências observadas podem ser representadas pela distribuição de probabilidades. A estatística de teste é obtida pela diferença máxima entre as funções de probabilidades acumuladas, empírica e teórica. O valor crítico do teste $\left(|\Delta F|_{t a b(n, \alpha)}\right)$ é obtido em função do nível de significância testado e do tamanho da amostra, o qual se rejeita a hipótese nula caso este valor supere o valor da estatística do teste $\left(|\Delta F|_{\max }\right)$ (Franco et al., 2014):

$$
|\delta F|_{\max } \leq|\delta F|_{\operatorname{tab}(n, \alpha)}
$$

Já o teste de $\chi^{2}$ agrupa os dados da série em classes de frequência e acumula os erros entre as frequências observadas e estimadas de cada classe. A estatística do teste é obtida por meio da tabela de $\chi^{2}$, adotando-se o valor tabelado em função do nível de significância e do grau de liberdade $\left(\chi_{t a b}^{2}\right)$. Trata-se de um teste quantitativo e considerado mais rigoroso em relação ao teste de KS. O calculo do $\chi^{2}$ é dado por (Franco et al., 2014; Caldeira et al., 2015):

$$
\chi^{2}=\sum_{i=1}^{n} \frac{\left(f_{o_{i}}-f_{t_{i}}\right)^{2}}{f_{t_{i}}}
$$

em que $\chi^{2}$ é a estatística do teste; $f_{o_{i}}$ é a frequência observada na i-ésima classe; $f_{t_{i}}$ é a frequência estimada na i-ésima classe.

Para o teste de $\chi^{2}$ rejeita-se a hipótese nula de que existe aderência da distribuição de probabilidades ao conjunto de dados, quando $\chi^{2}<\chi_{t a b}^{2}$.

$\mathrm{O}$ teste de Fi estima o coeficiente de correlação (r) entre as observações $\left(\mathrm{X}_{\mathrm{i}}\right)$ e os quantis teóricos $\left(W_{i}\right)$, os quais são calculados por $w i=F_{X}^{-1}\left(1-q_{i}\right)$, em que $F_{X}^{-1}$ a função inversa da fdp e $q_{i}$ representa a probabilidade empírica correspondente à ordem de classificação i. Quando $r_{F i}>r_{\text {tab }}$, admite-se que a amostra pode ser representada pela respectiva distribuição testada. Os valores críticos do teste de Fi são específicos para cada distribuição, enquanto a estatística do teste de Filliben é dada por 
(Junqueira Júnior, Mello e Alves, 2015):

$$
r_{F i}=\frac{\sum_{i=1}^{N}\left(X_{i}-\bar{X}\right)\left(W_{i}-\bar{W}\right)}{\sqrt{\sum_{i=1}^{N}\left(X_{i}-\bar{X}\right)^{2} \sum_{i=1}^{N}\left(W_{i}-\bar{W}\right)}}
$$

em que $r_{F i}$ é a estatpistica do teste; $X_{i}$ é o i-ésimo valor observado; $X$ é a média dos valores observados; $W_{i}$ é o iésimo valor estimado; $W$ é a média dos valores estimados.

$\mathrm{O}$ teste de $\mathrm{AD}$ procura ponderar de maneira mais efetiva as caudas das distribuições, devido à possibilidade de observações maiores ou menores da amostra em alterar a qualidade do ajuste. $\mathrm{O}$ teste baseia-se na diferença entre as funções de probabilidades acumuladas, empírica e teórica (Franco et al., 2014):

$$
A D^{2}=-N-\sum_{i=1}^{N} \frac{(2 i-1)[\ln (P 1)+\ln (P 2)]}{N}
$$

em que $A D^{2}$ é a estatística do teste; i é a ordem de cada elemento da série; $P 1$ é a probabilidade de não excedência calculada pela distribuição de probabilidade com os dados em ordem crescente; $P 2$ é a probabilidade excedência calculada pela distribuição de probabilidade com os dados em ordem decrescente; $N$ é o tamanho da amostra.

$\mathrm{O}$ valor calculado do teste deve ser comparado a um valor crítico $\mathrm{p}$, em função da distribuição de probabilidades e do tamanho da amostra. Se o valor calculado do teste $\left(\mathrm{AD}^{2}\right)$ for menor que o tabelado, admite-se que a distribuição de probabilidades se ajusta ao conjunto de dados observados. Como os valores críticos para a distribuição GEV são inexistentes, utilizaram-se os valores da distribuição Gumbel para testar a aderência desta distribuição, às séries de dados (Beskow et al., 2015).

A análise de incerteza foi realizada a partir da estimativa das precipitações máximas esperadas para os períodos de retorno de 2, 5, 10, 25, 50 e 100 anos (quantis notáveis), através do erro padrão da estimativa $\left(\mathrm{S}_{\mathrm{T}}\right)$ pertinente a cada estimativa ( Hosking, 1986; Kite, 1987; Naghettini e Pinto, 2007). Utilizou-se a média de $\mathrm{S}_{\mathrm{T}}$ para cada modelo probabilístico utilizado e para cada método de estimativa dos parâmetros, sendo o modelo com menor $\mathrm{S}_{\mathrm{T}}$ médio, considerado o de melhor desempenho.

Calcularam-se os erros de predição médios (epm) e as seguintes estatísticas de ajuste para comparar a frequência observada $\left(f_{\text {obs }}\right)$ e a frequência teórica de cada distribuição: coeficiente de correlação (r), raiz do quadrado médio do erro (rqme), índice de concordância de Willmott (d), índice BIAS e o índice c, de acordo com Martins et al. (2014). Realizou-se a comparação entre os diversos modelos de distribuição de probabilidades através do valor ponderado (vp) dos escores das estatísticas de ajuste. Foi atribuído o peso 1 para o modelo com melhor desem- penho, em cada estatística de ajuste, o peso 2 para o modelo com o segundo melhor desempenho, e assim sucessivamente para os demais modelos. O modelo que obtiver o menor somatório dos escores foi considerado o melhor modelo.

Por fim, foram verificados quais os testes de aderência apresentaram maior rigor em aceitar a hipótese de aderência e quais as distribuições de probabilidade e seus respectivos métodos de estimação dos parâmetros obtiveram melhor desempenho em cada um dos testes. Também foi verificado se um ou mais testes de aderência convergiram para a escolha da distribuição de probabilidade com melhor(s) desempenho(s) na análise de incertezas e/ou nas estatísticas de ajuste, para a tentativa de estabelecer um critério a respeito do método mais apropriado para a escolha da melhor distribuição de probabilidades.

\section{Resultados e Discussão}

Os testes de aderência apontaram diferentes resultados quanto à adequação das distribuições de probabilidade testadas, nos diferentes métodos de ajuste (Tabela 2). O teste de KS foi o menos rigoroso e admitiu aderência em todas as situações testadas. $O$ teste de $\chi^{2}$ rejeitou a hipótese de aderência das distribuições ao conjunto de dados em 5\% das situações, enquanto os testes de Fi e de AD rejeitaram a mesma hipótese em $41 \%$ e em $17 \%$ dos casos, respectivamente. $\mathrm{O}$ teste de $\mathrm{Fi}$ mostrou-se mais rigoroso quanto a admitir a aderência da distribuição ao conjunto de dados.

Junqueira Júnior et al. (2015) testaram a aderência das distribuições Gumbel e GEV em estações na bacia hidrográfica do rio Doce, pelos testes de $\chi^{2}$ e Fi, para diferentes métodos de ajuste das distribuições (MM, ML e $\mathrm{MV}$ ). Os autores verificaram maior rigor do teste de Fi, apenas para o método da máxima verossimilhança, enquanto que o teste $\chi^{2}$ foi mais rigoroso para os ajustes das distribuições pelos métodos dos momentos e momentos-L. Beskow et al. (2015) utilizou os mesmos testes utilizados neste estudo e verificou que o teste de AD e o teste de Fi foram os mais rigorosos em relação aos testes de $\chi^{2} \mathrm{e}$ KS. Shin et al. (2012) constataram maior rigor no teste de $\mathrm{AD}$ em relação aos testes de $\chi^{2}$, KS e Cramer Von Mises. Esses resultados demonstram que, a exemplo do observado no presente trabalho, os testes de Fi e AD tendem a ser mais rigorosos que os testes de $\chi^{2}$ e KS. Porém, um maior rigor na aceitação da hipótese da aderência pode não significar a melhor eficiência no ajuste da fdp ao conjunto de dados, o que justifica a verificação quanto à convergência dos testes de aderência à distribuição com melhor desempenho na análise de incerteza e com melhor desempenho nas estatísticas de ajuste.

A exemplo do apresentado nos resultados deste trabalho, o teste de KS mostrou-se menos rigoroso em 
Tabela 2 - Estatísticas dos testes de aderência, erro padrão da estimativa $\left(\mathrm{S}_{\mathrm{T}}\right)$ e estatísticas de ajuste das funções densidade de probabilidade aos conjuntos de dados.

\begin{tabular}{|c|c|c|c|c|c|c|c|c|c|c|c|c|c|}
\hline \multirow[t]{2}{*}{ Estação } & \multirow[t]{2}{*}{ Dist. } & \multirow[t]{2}{*}{ Mét. } & \multicolumn{4}{|c|}{ Estatísticas dos testes de aderência } & \multirow[t]{2}{*}{$\mathrm{S}_{\mathrm{T}}(\mathrm{mm})$} & \multicolumn{6}{|c|}{ Estatísticas de ajuste } \\
\hline & & & $\overline{\mathrm{KS}}$ & $\chi^{2}$ & $\mathrm{r}_{\mathrm{Fi}}$ & $\mathrm{AD}^{2}$ & & $\overline{E p m(\%)}$ & $\mathrm{r}$ & BIAS (mm) & rqme $(\mathrm{mm})$ & $\mathrm{d}$ & $\mathrm{c}$ \\
\hline \multirow[t]{8}{*}{ Baependi } & \multirow[t]{3}{*}{ GEV } & MM & 0,15 & 8,98 & $0,91^{*}$ & 0,61 & 13,8 & 0,35 & 0,981 & 0,030 & 0,34 & 0,98 & 0,96 \\
\hline & & ML & 0,12 & 9,50 & $0,90^{*}$ & 0,49 & 10,7 & 0,47 & 0,825 & 0,356 & 0,45 & 0,98 & 0,81 \\
\hline & & MV & 0,12 & 8,97 & $0,90^{*}$ & 0,52 & 10,5 & 0,35 & 0,984 & 0,009 & 0,34 & 0,98 & 0,96 \\
\hline & \multirow[t]{3}{*}{ GUM } & MM & 0,14 & 8,94 & 0,97 & 0,59 & 11,1 & 0,35 & 0,981 & 0,028 & 0,34 & 0,98 & 0,96 \\
\hline & & $\mathrm{ML}$ & 0,13 & 8,93 & 0,97 & 0,59 & 9,9 & 0,35 & 0,982 & 0,025 & 0,34 & 0,98 & 0,96 \\
\hline & & MV & 0,13 & 8,81 & 0,97 & 0,58 & 9,6 & 0,35 & 0,982 & 0,016 & 0,34 & 0,98 & 0,96 \\
\hline & \multirow[t]{2}{*}{ LN2 } & $\mathrm{MM}$ & 0,14 & 9,11 & $0,92^{*}$ & 0,77 & 10,5 & 0,37 & 0,986 & $-0,018$ & 0,36 & 0,98 & 0,96 \\
\hline & & MV & 0,13 & 9,07 & $0,74^{*}$ & 0,48 & 10,2 & 0,38 & 0,986 & $-0,032$ & 0,36 & 0,98 & 0,96 \\
\hline \multirow[t]{8}{*}{ Bairro Santa Cruz } & \multirow[t]{3}{*}{ GEV } & $\mathrm{MM}$ & 0,13 & 4,61 & 0,97 & 0,34 & 11,4 & 0,44 & 0,988 & $-0,018$ & 0,42 & 0,96 & 0,95 \\
\hline & & ML & 0,11 & 4,91 & 0,97 & 0,28 & 16,2 & 0,58 & 0,825 & 0,499 & 0,56 & 0,97 & 0,80 \\
\hline & & MV & 0,10 & 5,50 & 0,97 & 0,32 & 20,5 & 0,45 & 0,991 & 0,004 & 0,43 & 0,96 & 0,96 \\
\hline & \multirow[t]{3}{*}{ GUM } & $\mathrm{MM}$ & 0,11 & 5,14 & 0,98 & 0,31 & 12,5 & 0,45 & 0,991 & $-0,006$ & 0,43 & 0,96 & 0,95 \\
\hline & & ML & 0,11 & 4,84 & 0,98 & 0,28 & 12,1 & 0,44 & 0,991 & 0,002 & 0,42 & 0,96 & 0,95 \\
\hline & & MV & 0,11 & 5,39 & 0,98 & 0,33 & 10,4 & 0,45 & 0,991 & $-0,002$ & 0,43 & 0,96 & 0,95 \\
\hline & \multirow[t]{2}{*}{ LN2 } & MM & 0,11 & 5,81 & 0,96 & 0,42 & 15,4 & 0,46 & 0,990 & $-0,004$ & 0,44 & 0,96 & 0,95 \\
\hline & & MV & 0,10 & 5,46 & $0,72^{*}$ & 0,02 & 15,9 & 0,44 & 0,989 & 0,013 & 0,42 & 0,96 & 0,95 \\
\hline \multirow[t]{8}{*}{ Caxambu } & \multirow[t]{3}{*}{ GEV } & MM & 0,12 & 6,00 & $0,93^{*}$ & 0,53 & 8,3 & 0,43 & 0,989 & 0,026 & 0,42 & 0,98 & 0,97 \\
\hline & & $\mathrm{ML}$ & 0,08 & 7,58 & $0,93^{*}$ & 0,39 & 6,7 & 0,43 & 0,993 & 0,001 & 0,41 & 0,98 & 0,97 \\
\hline & & MV & 0,10 & 5,66 & $0,93^{*}$ & 0,44 & 7,1 & 0,42 & 0,991 & 0,011 & 0,41 & 0,98 & 0,97 \\
\hline & \multirow[t]{3}{*}{ GUM } & $\mathrm{MM}$ & 0,12 & 6,20 & 0,97 & 0,64 & 7,9 & 0,43 & 0,988 & 0,032 & 0,42 & 0,98 & 0,97 \\
\hline & & ML & 0,12 & 6,20 & 0,97 & 0,64 & 7,2 & 0,43 & 0,988 & 0,032 & 0,42 & 0,98 & 0,97 \\
\hline & & MV & 0,11 & 5,79 & 0,97 & 0,58 & 7,2 & 0,42 & 0,988 & 0,022 & 0,41 & 0,98 & 0,97 \\
\hline & \multirow[t]{2}{*}{ LN2 } & MM & 0,10 & 5,74 & $0,93^{*}$ & $1,01^{*}$ & 6,9 & 0,43 & 0,991 & 0,019 & 0,42 & 0,98 & 0,97 \\
\hline & & MV & 0,10 & 5,73 & $0,65^{*}$ & 0,63 & 6,9 & 0,43 & 0,990 & 0,019 & 0,42 & 0,98 & 0,97 \\
\hline \multirow[t]{8}{*}{ Conceição dos Ouros } & GEV & $\mathrm{MM}$ & 0,07 & 0,81 & $0,96^{*}$ & $0,21^{*}$ & 7,8 & 0,39 & 0,996 & 0,003 & 0,38 & 0,99 & 0,98 \\
\hline & & $\mathrm{ML}$ & 0,06 & 0,97 & $0,96^{*}$ & $0,21^{*}$ & 8,4 & 0,39 & 0,996 & 0,002 & 0,38 & 0,99 & 0,98 \\
\hline & & MV & 0,07 & 0,87 & $0,96^{*}$ & $0,21^{*}$ & 8,1 & 0,39 & 0,996 & 0,002 & 0,38 & 0,99 & 0,98 \\
\hline & GUM & $\mathrm{MM}$ & 0,09 & $0,45^{*}$ & 0,99 & $0,35^{*}$ & 8,6 & 0,40 & 0,996 & 0,016 & 0,39 & 0,99 & 0,98 \\
\hline & & $\mathrm{ML}$ & 0,08 & $0,48^{*}$ & 0,99 & $0,31^{*}$ & 8,1 & 0,39 & 0,996 & 0,020 & 0,38 & 0,99 & 0,98 \\
\hline & & MV & 0,07 & $0,62^{*}$ & 0,99 & $0,28^{*}$ & 7,8 & 0,39 & 0,996 & 0,013 & 0,38 & 0,99 & 0,98 \\
\hline & LN2 & $\mathrm{MM}$ & 0,10 & $0,48^{*}$ & $0,95^{*}$ & $0,42^{*}$ & 9,7 & 0,40 & 0,995 & 0,022 & 0,39 & 0,99 & 0,98 \\
\hline & & MV & 0,09 & 1,28 & $0,74^{*}$ & 0,68 & 12,0 & 0,38 & 0,993 & 0,039 & 0,37 & 0,99 & 0,98 \\
\hline Fazenda Juca & GEV & $\mathrm{MM}$ & 0,08 & 1,87 & 0,98 & 0,22 & 9,5 & 0,40 & 0,996 & $-0,014$ & 0,38 & 0,98 & 0,98 \\
\hline Casemiro & & ML & 0,07 & 0,77 & 0,99 & 0,17 & 12,7 & 0,40 & 0,997 & 0,002 & 0,38 & 0,98 & 0,98 \\
\hline & & MV & 0,08 & 1,72 & 0,99 & 0,21 & 14,5 & 0,40 & 0,997 & 0,001 & 0,39 & 0,98 & 0,98 \\
\hline & GUM & $\mathrm{MM}$ & 0,08 & 1,79 & 0,99 & 0,23 & 10,9 & 0,41 & 0,996 & $-0,001$ & 0,39 & 0,98 & 0,98 \\
\hline & & $\mathrm{ML}$ & 0,06 & 1,39 & 0,99 & 0,17 & 10,6 & 0,40 & 0,997 & 0,007 & 0,39 & 0,98 & 0,98 \\
\hline & & MV & 0,07 & 1,64 & 0,99 & 0,20 & 9,3 & 0,40 & 0,997 & 0,004 & 0,39 & 0,98 & 0,98 \\
\hline & LN2 & MM & 0,08 & 1,65 & 0,97 & 0,38 & 10,2 & 0,40 & 0,997 & $-0,006$ & 0,39 & 0,98 & 0,98 \\
\hline & & MV & 0,08 & 1,65 & $0,48^{*}$ & 0,44 & 10,1 & 0,40 & 0,997 & $-0,004$ & 0,39 & 0,98 & 0,98 \\
\hline Ilicínea & GEV & $\mathrm{MM}$ & 0,14 & 1,56 & 0,97 & 0,37 & 16,8 & 0,43 & 0,983 & $-0,026$ & 0,40 & 0,95 & 0,94 \\
\hline & & $\mathrm{ML}$ & 0,12 & 1,58 & 0,97 & 0,37 & 28,0 & 0,44 & 0,993 & 0,014 & 0,41 & 0,95 & 0,95 \\
\hline & & MV & 0,11 & 1,71 & 0,97 & 0,19 & 30,7 & 0,45 & 0,993 & 0,002 & 0,42 & 0,95 & 0,95 \\
\hline & GUM & $\mathrm{MM}$ & 0,14 & 1,57 & 0,97 & 0,33 & 14,7 & 0,43 & 0,985 & $-0,022$ & 0,41 & 0,95 & 0,94 \\
\hline & & ML & 0,14 & 1,54 & 0,97 & 0,33 & 13,7 & 0,43 & 0,985 & $-0,019$ & 0,40 & 0,95 & 0,94 \\
\hline
\end{tabular}


Tabela 2 - continued

\begin{tabular}{|c|c|c|c|c|c|c|c|c|c|c|c|c|c|}
\hline \multirow[t]{2}{*}{ Estação } & \multirow[t]{2}{*}{ Dist. } & \multirow[t]{2}{*}{ Mét. } & \multicolumn{4}{|c|}{ Estatísticas dos testes de aderência } & \multirow[t]{2}{*}{$\mathrm{S}_{\mathrm{T}}(\mathrm{mm})$} & \multicolumn{6}{|c|}{ Estatísticas de ajuste } \\
\hline & & & $\overline{\mathrm{KS}}$ & $\chi^{2}$ & $\mathrm{r}_{\mathrm{Fi}}$ & $\mathrm{AD}^{2}$ & & Epm (\%) & $\mathrm{r}$ & BIAS (mm) & rqme $(\mathrm{mm})$ & $\mathrm{d}$ & $\mathrm{c}$ \\
\hline \multirow{11}{*}{ Monsenhor Paulo } & \multirow{3}{*}{$\mathrm{LN} 2$} & MV & 0,14 & 2,08 & 0,97 & 0,35 & 10,8 & 0,45 & 0,988 & $-0,029$ & 0,42 & 0,95 & $\overline{0,94}$ \\
\hline & & $\mathrm{MM}$ & 0,15 & 1,76 & 0,97 & 0,57 & 13,1 & 0,43 & 0,981 & $-0,036$ & 0,41 & 0,95 & 0,94 \\
\hline & & MV & 0,16 & 2,08 & $0,57^{*}$ & 0,69 & 11,5 & 0,44 & 0,982 & $-0,047$ & 0,41 & 0,95 & 0,94 \\
\hline & \multirow[t]{3}{*}{ GEV } & $\mathrm{MM}$ & 0,12 & 1,51 & 0,96 & 0,38 & 11,3 & 0,34 & 0,991 & $-0,011$ & 0,33 & 0,99 & 0,98 \\
\hline & & $\mathrm{ML}$ & 0,11 & 1,33 & 0,97 & 0,35 & 14,2 & 0,34 & 0,991 & 0,001 & 0,33 & 0,99 & 0,98 \\
\hline & & MV & 0,11 & 1,59 & 0,97 & 0,40 & 17,1 & 0,35 & 0,991 & 0,004 & 0,34 & 0,99 & 0,98 \\
\hline & \multirow[t]{3}{*}{ GUM } & $\mathrm{MM}$ & 0,12 & 1,58 & 0,99 & 0,39 & 11,6 & 0,34 & 0,991 & $-0,004$ & 0,34 & 0,99 & 0,98 \\
\hline & & ML & 0,11 & 1,33 & 0,99 & 0,35 & 11,0 & 0,34 & 0,991 & 0,001 & 0,33 & 0,99 & 0,98 \\
\hline & & MV & 0,12 & 1,71 & 0,99 & 0,40 & 9,6 & 0,35 & 0,991 & 0,000 & 0,34 & 0,99 & 0,98 \\
\hline & \multirow[t]{2}{*}{ LN2 } & $\mathrm{MM}$ & 0,11 & 1,44 & $0,95^{*}$ & 0,43 & 11,4 & 0,35 & 0,991 & $-0,005$ & 0,34 & 0,99 & 0,98 \\
\hline & & MV & 0,11 & 1,45 & $0,62^{*}$ & 0,49 & 11,7 & 0,34 & 0,991 & $-0,003$ & 0,33 & 0,99 & 0,98 \\
\hline \multirow[t]{8}{*}{ Pouso Alto } & \multirow[t]{3}{*}{ GEV } & $\mathrm{MM}$ & 0,06 & 3,25 & 0,97 & 0,14 & 9,9 & 0,39 & 0,997 & $-0,006$ & 0,38 & 0,98 & 0,98 \\
\hline & & $\mathrm{ML}$ & 0,06 & 3,24 & 0,97 & 0,12 & 12,4 & 0,39 & 0,998 & 0,006 & 0,38 & 0,98 & 0,98 \\
\hline & & MV & 0,05 & 3,32 & 0,97 & 0,12 & 11,4 & 0,39 & 0,998 & $-0,002$ & 0,38 & 0,98 & 0,98 \\
\hline & \multirow[t]{3}{*}{ GUM } & $\mathrm{MM}$ & 0,05 & 3,31 & 0,99 & 0,13 & 10,4 & 0,39 & 0,998 & 0,004 & 0,38 & 0,98 & 0,98 \\
\hline & & ML & 0,06 & 3,27 & 0,99 & 0,13 & 9,8 & 0,39 & 0,998 & 0,009 & 0,38 & 0,98 & 0,98 \\
\hline & & MV & 0,06 & 3,21 & 0,99 & 0,12 & 8,9 & 0,39 & 0,998 & 0,004 & 0,38 & 0,98 & 0,98 \\
\hline & \multirow[t]{2}{*}{ LN2 } & MM & 0,06 & 3,34 & $0,96^{*}$ & 0,33 & 9,7 & 0,39 & 0,998 & $-0,002$ & 0,38 & 0,98 & 0,98 \\
\hline & & MV & 0,06 & 3,34 & $0,57^{*}$ & 0,25 & 9,5 & 0,39 & 0,998 & 0,000 & 0,38 & 0,98 & 0,98 \\
\hline \multirow[t]{8}{*}{ Três Corações } & \multirow[t]{3}{*}{ GEV } & MM & 0,12 & 0,69 & $0,97^{*}$ & 0,33 & 6,8 & 0,45 & 0,991 & $-0,002$ & 0,44 & 0,97 & 0,96 \\
\hline & & $\mathrm{ML}$ & 0,12 & 0,72 & $0,97^{*}$ & 0,32 & 7,8 & 0,45 & 0,991 & 0,004 & 0,44 & 0,97 & 0,96 \\
\hline & & MV & 0,13 & 0,58 & $0,97^{*}$ & 0,34 & 6,5 & 0,45 & 0,990 & $-0,007$ & 0,44 & 0,97 & 0,96 \\
\hline & \multirow[t]{3}{*}{ GUM } & MM & 0,12 & 2,32 & 0,98 & 0,60 & 9,4 & 0,46 & 0,989 & 0,035 & 0,45 & 0,97 & 0,96 \\
\hline & & ML & 0,12 & 1,99 & 0,98 & 0,54 & 9,1 & 0,46 & 0,988 & 0,039 & 0,44 & 0,97 & 0,96 \\
\hline & & MV & 0,13 & 1,96 & 0,98 & 0,52 & 9,6 & 0,44 & 0,988 & 0,022 & 0,43 & 0,97 & 0,96 \\
\hline & \multirow[t]{2}{*}{ LN2 } & $\mathrm{MM}$ & 0,10 & 1,10 & 0,97 & 0,93 & 7,9 & 0,46 & 0,991 & 0,020 & 0,44 & 0,97 & 0,96 \\
\hline & & MV & 0,11 & 1,26 & $0,43^{*}$ & 0,41 & 8,6 & 0,45 & 0,990 & 0,024 & 0,43 & 0,97 & 0,96 \\
\hline \multirow{8}{*}{$\begin{array}{l}\text { UHE Marechal Mas- } \\
\text { carenhas de Moraes } \\
\text { rio Sapucaí }\end{array}$} & GEV & MM & 0,13 & 6,62 & $0,90^{*}$ & 0,49 & 15,0 & 0,45 & 0,981 & 0,019 & 0,43 & 0,97 & 0,95 \\
\hline & & ML & 0,13 & 7,06 & $0,90^{*}$ & 0,47 & 12,6 & 0,46 & 0,982 & 0,009 & 0,44 & 0,97 & 0,95 \\
\hline & & MV & 0,12 & 7,10 & $0,90^{*}$ & 0,48 & 10,8 & 0,46 & 0,982 & 0,009 & 0,44 & 0,97 & 0,95 \\
\hline & GUM & $\mathrm{MM}$ & 0,15 & 7,79 & 0,94 & 0,58 & 15,0 & 0,44 & 0,984 & 0,004 & 0,42 & 0,97 & 0,95 \\
\hline & & ML & 0,14 & 8,51 & 0,94 & 0,47 & 12,6 & 0,45 & 0,985 & $-0,009$ & 0,43 & 0,97 & 0,95 \\
\hline & & MV & 0,13 & 10,58 & 0,94 & 0,45 & 10,8 & 0,46 & 0,986 & $-0,006$ & 0,44 & 0,97 & 0,95 \\
\hline & LN2 & $\mathrm{MM}$ & 0,16 & 7,28 & $0,88^{*}$ & 0,64 & 15,0 & 0,44 & 0,984 & 0,001 & 0,42 & 0,97 & 0,95 \\
\hline & & MV & 0,14 & 11,38 & $0,82^{*}$ & 0,63 & 12,1 & 0,45 & 0,986 & $-0,014$ & 0,43 & 0,97 & 0,95 \\
\hline Usina do Chicão & GEV & $\mathrm{MM}$ & 0,15 & 19,55 & $0,91^{*}$ & $1,15^{*}$ & 10,0 & 0,34 & 0,973 & 0,019 & 0,33 & 0,99 & 0,96 \\
\hline & & $\mathrm{ML}$ & 0,14 & 20,22 & $0,91^{*}$ & $1,13^{*}$ & 8,6 & 0,34 & 0,976 & $-0,004$ & 0,33 & 0,99 & 0,96 \\
\hline & & MV & 0,15 & 19,36 & $0,91^{*}$ & $1,13^{*}$ & 8,9 & 0,33 & 0,973 & 0,002 & 0,32 & 0,99 & 0,96 \\
\hline & GUM & $\mathrm{MM}$ & 0,17 & 22,78 & 0,96 & $1,49^{*}$ & 11,5 & 0,45 & 0,975 & 0,473 & 0,32 & 0,98 & 0,96 \\
\hline & & $\mathrm{ML}$ & 0,17 & 23,60 & 0,96 & $1,52^{*}$ & 10,4 & 0,45 & 0,977 & 0,472 & 0,32 & 0,98 & 0,96 \\
\hline & & MV & 0,17 & 21,34 & 0,96 & $1,43^{*}$ & 11,4 & 0,43 & 0,982 & 0,462 & 0,30 & 0,98 & 0,96 \\
\hline & LN2 & MM & 0,18 & 24,61 & $0,94^{*}$ & $1,71^{*}$ & 11,7 & 0,45 & 0,971 & 0,473 & 0,32 & 0,98 & 0,95 \\
\hline & & MV & 0,20 & 23,98 & $0,71^{*}$ & 0,51 & 15,2 & 0,43 & 0,975 & 0,481 & 0,31 & 0,98 & 0,95 \\
\hline
\end{tabular}

*Distribuição não adequada para representar os dados observados pelo respectivo teste de aderência, com significância de 5\%. 
diversos estudos (Franco et al., 2014; Beskow et al., 2015). Por se tratar de um teste qualitativo, sugere-se que não é possível utilizá-lo como parâmetro comparativo do ajuste entre diferentes distribuições de probabilidade (Caldeira et al., 2015). Apesar disso, devido a sua simplicidade, o teste de KS tem larga utilização na literatura, especialmente em estudos de precipitações máximas (Junqueira Júnior et al., 2007; Vitor et al., 2013; Pereira et al., 2014). Poucos estudos, especialmente no Brasil, utilizam os testes de $\chi^{2}$, Fi e de $\mathrm{AD}$, provavelmente devido a algumas circunstâncias, as quais se destacam: o teste de $\chi^{2}$ pode ser dividido em três tipos (teste de adequação do ajustamento, teste de aderência e teste de independência), o que acaba por espargir a sua função de aderência; os testes de Fi e de AD possuem os valores críticos determinados somente para algumas distribuições, o que limita a sua utilização comparar a estatística dos respectivos testes (Naghettini e Pinto, 2007; Beskow et al., 2015).

Uma questão interessante a ser ressaltada foi o desempenho da distribuição Gumbel no teste de Fi. Em nenhuma estação a aderência da distribuição ao conjunto de dados observados foi rejeitada e as estatísticas do teste entre os diferentes métodos de ajuste da distribuição Gumbel (MM, ML e MV) foram iguais. Tal situação foi semelhante à observada também em estudo de eventos extremos na região do alto do rio Grande (Junqueira Júnior et al., 2015), próxima à bacia do rio Sapucaí. Outros estudos também constataram que o teste de Fi afeta menos a aderência da distribuição Gumbel ao conjunto de dados, do que as demais distribuições, independente do método de ajuste dos parâmetros (Franco et al., 2014; Junqueira Júnior, Mello e Alves, 2015). Uma possível explicação é a proximidade dos ajustes da distribuição Gumbel pelos diferentes métodos de estimação dos parâmetros, evidenciados pelos valores das estatísticas de ajuste semelhantes, especialmente o coeficiente de correlação entre a frequência observada e teórica, que é utilizado no cálculo da estatística do teste Fi.

As estatísticas de ajuste obtidas também estão apresentadas na Tabela 2. De maneira geral, os baixos valores de epm e de rqme indicam erros baixos. Os valores de $r$ indicam o grau de associação entre as frequências observada e estimada, sendo fortes (valores entre 0,70 e 0,89 ) ou muito fortes (valores entre 0,90 e 1.00). Os valores de BIAS indicam a tendência dos modelos (valores próximos à zero) em representar os dados observados (sem superestimar ou subestimar) e o desempenho da maioria dos modelos pode ser considerado ótimo devido aos altos valores das estatísticas d (superiores a 0,95). De acordo com a classificação do desempenho dos modelos pelo índice c, os ajustes podem ser considerados muito bons (valores entre 0,76 e 0,85 ) ou ótimos (superiores a 0,85 ).

A média dos $\mathrm{S}_{\mathrm{T}}$ provenientes da estimativa dos quantis de interesse também são considerados aceitáveis para estudos de eventos extremos de precipitação $\left(\mathrm{S}_{\mathrm{T}}\right.$ médio máximo inferior a $31 \mathrm{~mm}$ ). A Fig. 2 mostra os valores das precipitações esperadas para os TR de 2, 5, 10, 25, 50 e 100 anos (quantis de interesse) e os respectivos $\mathrm{S}_{\mathrm{T}}$.Percebe-se que para a maioria das estações $(42 \%$ dos casos) a distribuição Gumbel com os parâmetros estimados pelo método MV obteve os menores $\mathrm{S}_{\mathrm{T}}$, seguida pela distribuição GEV com os parâmetros estimados também pelo método da MV (18\% dos casos). Esse fato comprova a importância dessas distribuições de probabilidade em representar os dados observados de precipitações diárias máximas anuais.

$\mathrm{O}$ teste de aderência que apresentou o maior número de convergências para a escolha da distribuição de probabilidades com melhor desempenho na análise de incertezas $\left(\mathrm{S}_{\mathrm{T}}\right)$ foi o teste de $\mathrm{Fi}$, em $64 \%$ das situações. Os demais testes convergiram para a escolha da distribuição em $27 \%$ das situações, quando se considera a análise de incertezas. Quando se considera as estatísticas de ajuste, o teste de Fi também apresentou vantagens em relação aos testes de $\mathrm{KS}, \chi^{2}$ e AD, com convergência para a escolha da distribuição com melhor desempenho em $45 \%$ das situações, seguido do teste de $\chi^{2}$, que coincidiu em $18 \%$ dos casos. Os testes de KS e $\mathrm{AD}$ não convergiram em nenhuma estação para a escolha da fdp com melhor desempenho nas estatísticas de ajuste (Fig. 3).

Houve similaridade estatística no desempenho das distribuições GUM e GEV em três situações: no teste de $\chi^{2}$ e no teste de AD houve similaridade estatística nos ajustes destas fdp ajustadas pelo método ML e na análise de incerteza houve similaridade estatística no ajuste pelo método MV. No teste de Fi, houve similaridade estatística de desempenho da distribuição GUM, ajustada pelos métodos MM, ML e MV para a maioria das situações (em 10 estações). Nesses casos, as distribuições com similaridade estatística foram consideradas a de melhor desempenho simultaneamente.

Como o desempenho das distribuições probabilísticas para o $\mathrm{S}_{\mathrm{T}}$ e para as estatísticas de ajuste foram próximas, principalmente quando se considera os três melhores desempenhos, considerou-se a convergência dos testes de aderência na indicação da distribuição de probabilidades entre os três melhores desempenhos. $\mathrm{O}$ teste de Fi continuou a apresentar maior número de indicações da fdp com melhor desempenho, tanto na análise de incertezas ( $91 \%$ doas situações) quanto no desempenho nas estatísticas de ajuste ( $100 \%$ das situações). O teste de $\chi^{2}$ e AD indicaram a melhor fdp na análise de incertezas em 55\% das situações, enquanto o teste de KS indicou a melhor distribuição em apenas $36 \%$ das situações. Já para o desempenho nas estatísticas de ajuste, o teste de $\chi^{2}$ apresentou número de convergências para escolha da distribuição de probabilidades em $73 \%$ dos casos, seguido dos testes de AD e KS, com convergências de $55 \%$ e $45 \%$, respectivamente (Fig. 4). Percebe-se que o teste de Fi, além de apresentar maior rigor na aderência da fdp ao 

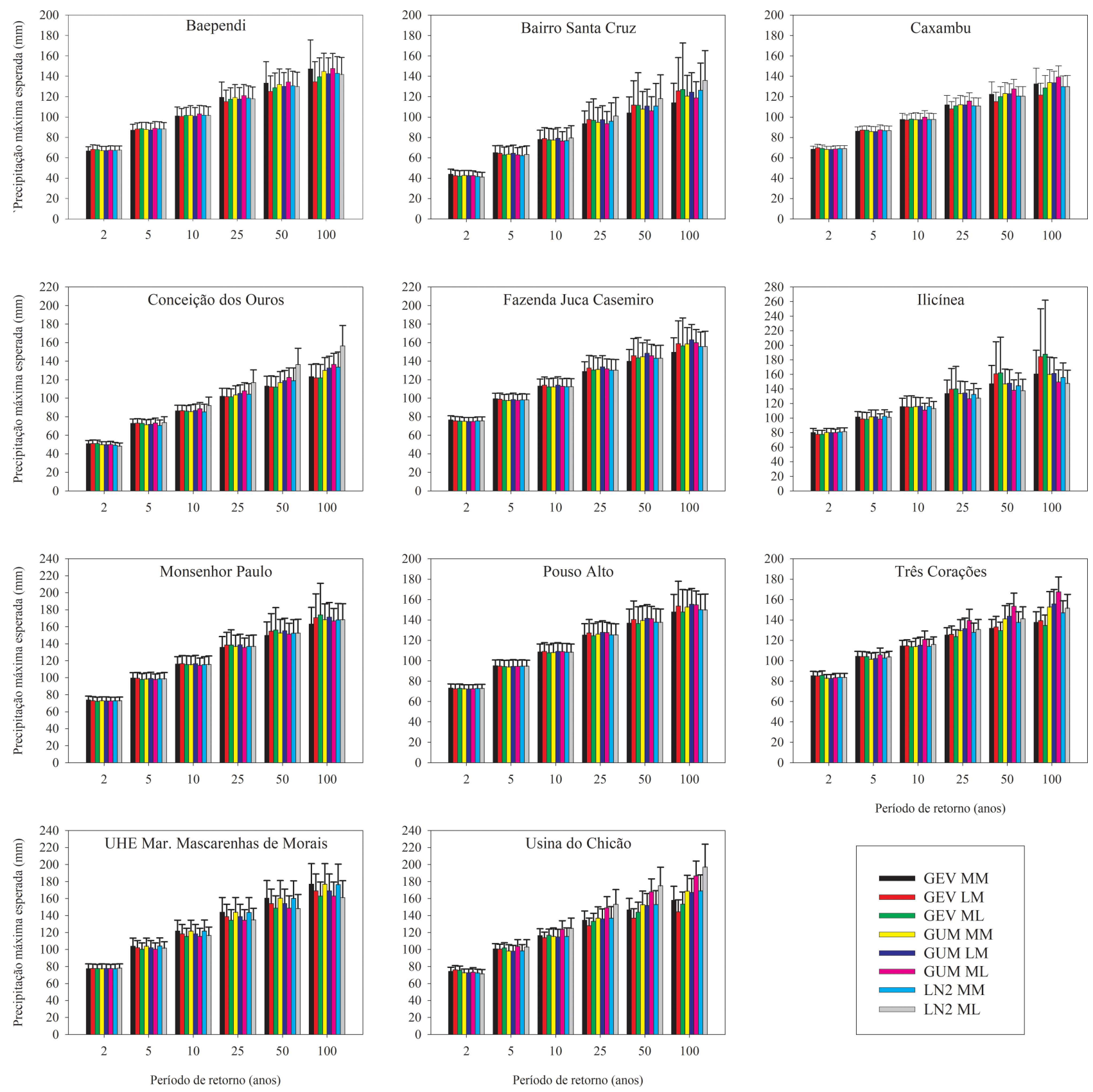

Figura 2 - Valores de precipitação máxima esperada $(\mathrm{mm})$ e seus respectivos erros padrão da estimativa $\left(\mathrm{S}_{\mathrm{T}}\right)$.

conjunto de dados, tende a coincidir com a distribuição de melhor desempenho na análise de incertezas e estatísticas de ajuste, devendo ser considerado sempre que possível na escolha de distribuições de probabilidade.

Deve-se ressaltar que a análise de incerteza, a partir do $\mathrm{S}_{\mathrm{T}}$, considera apenas os erros oriundos do processo de estimativa dos quantis de interesse, não considerando a aderência da distribuição à série de dados (Naghettini e Pinto, 2007). Logo, a escolha de uma fdp adequada é feita anteriormente à análise de incertezas. Em algumas ocasiões, os melhores desempenhos no $\mathrm{S}_{\mathrm{T}}$ ou nas estatísticas de ajuste foram de distribuições não aderentes à série de dados em alguns testes, especialmente o teste de Fi. Portanto, nem sempre o uso do teste de aderência mais rigoroso confere a escolha de distribuições de probabilidade com melhores estimativas de quantis de interesse. Nesse contexto, os testes de $\chi^{2}$ e AD tornam-se alternativas interessantes como testes de aderência de fdp ao conjunto de dados. 

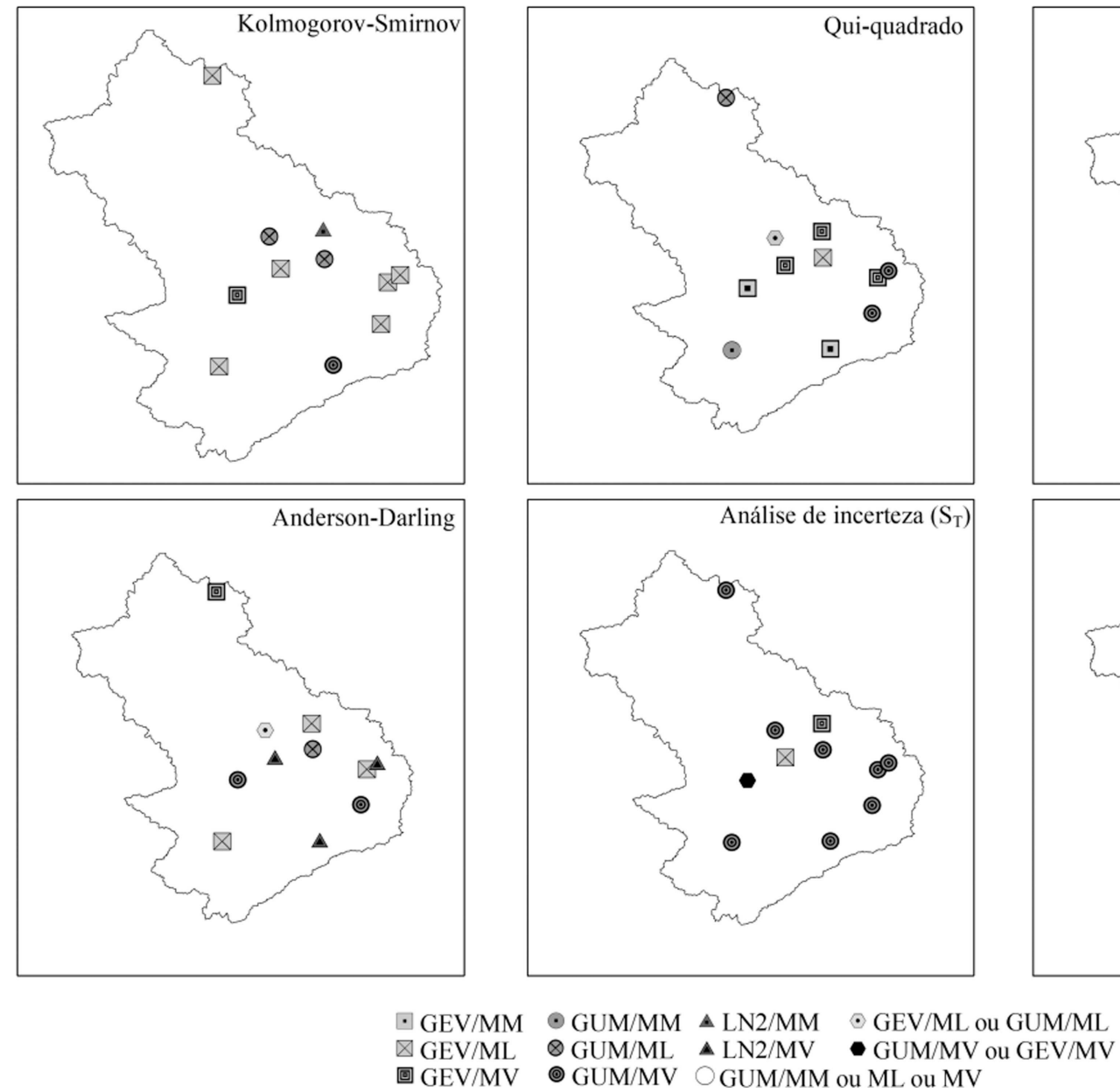

Figura 3 - Modelos probabilísticos com melhor desempenho de acordo com testes de aderência, estatísticas de ajuste e erro padrão da estimativa.>eak/ >*Círculos sem preenchimento de cor indicam similaridade estatística da distribuição Gumbel ajustada pelo MM, LM e MV.

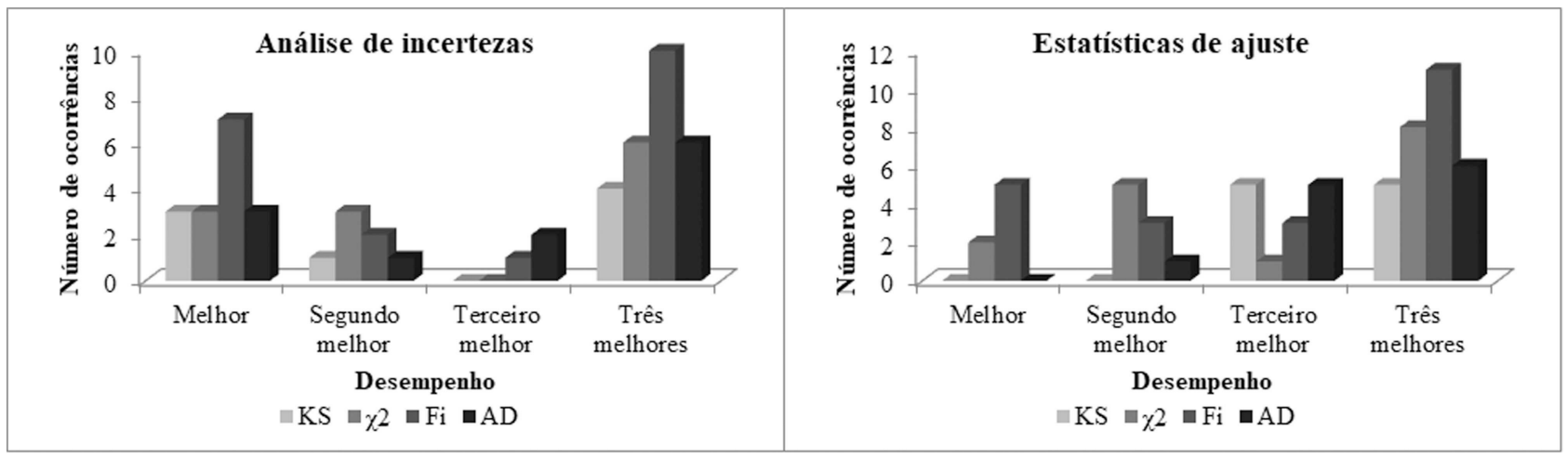

Figura 4 - Número de convergências na indicação de uma fdp entre os testes de aderência e a análise de incertezas (painel A) e entre os testes de aderência e as estatísticas de ajuste (painel B).

Os ajustes dos modelos probabilísticos podem ser observados na Fig. 5. De maneira geral, percebe-se bom ajuste dos modelos aos dados observados das diferentes estações e, inclusive, uma proximidade no comportamento das curvas dos diferentes modelos. Porém, observa-se um distanciamento das curvas ao conjunto de dados, especialmente nas estações com grande quantidade de rejeições da hipótese de aderência (UHE Marechal Mascarenhas de Moraes Rio Sapucaí e Usina do Chicão, por exemplo). 

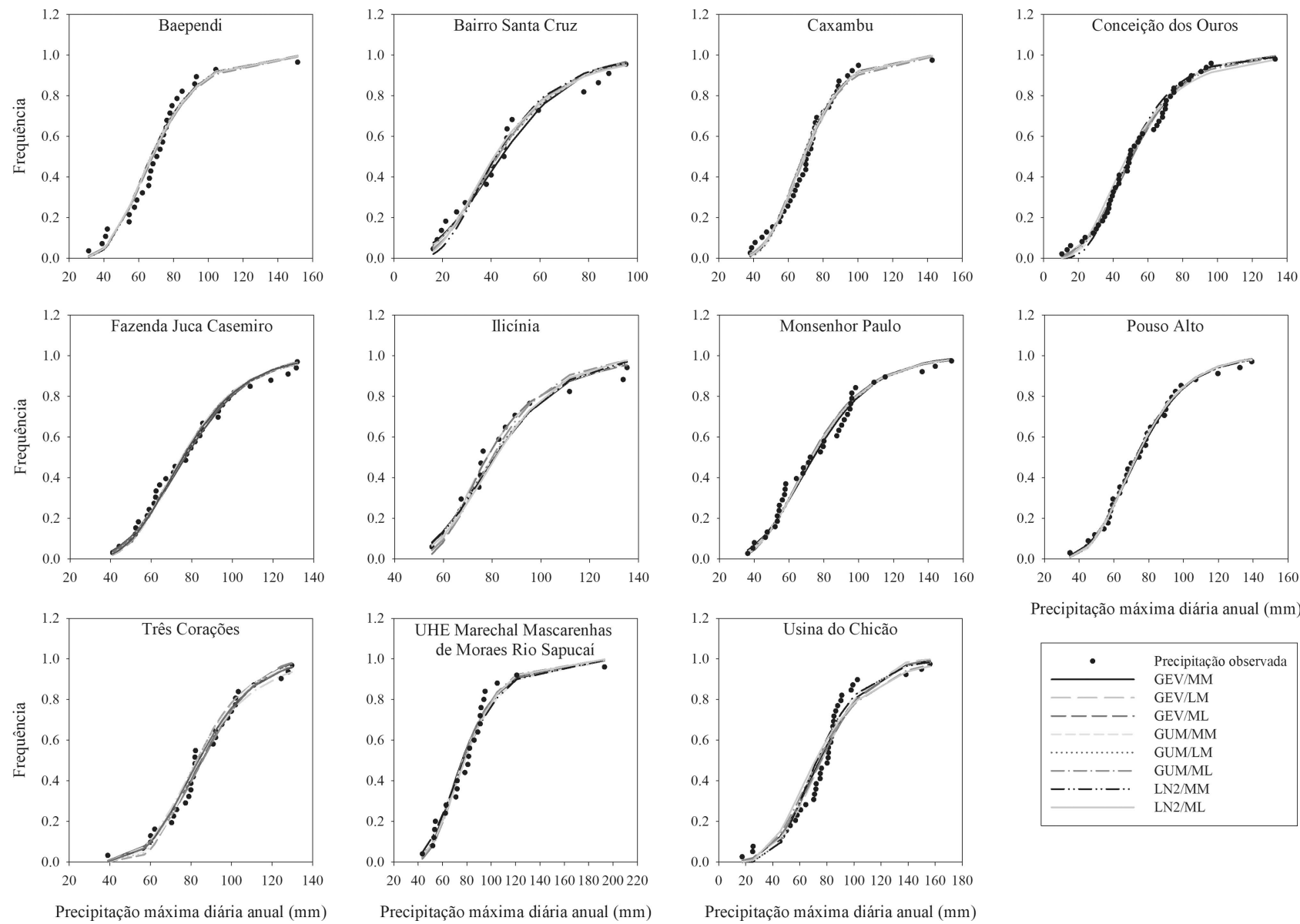

Precipitação máxima diária anual (mm)

Precipitação máxima diária anual (mm)

Precipitação máxima diária anual $(\mathrm{mm})$

Precipitação máxima diária anual $(\mathrm{mm})$

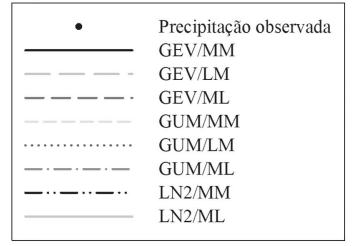

Figura 5 - Ajuste dos modelos probabilísticos à precipitação máxima diária anual de estações pluviométricas da bacia do rio Sapucaí, MG.

Apesar dos bons ajustes, o modelo probabilístico GEV, ajustado pelos métodos ML e MV, e o modelo GUM, com os parâmetros estimados pelo método MV, foram os que obtiveram mais indicações como a fdp mais adequada em representar os dados observados (Tabela 3). Esses resultados corroboram aos obtidos por Junqueira Júnior et al. (2015), que verificaram a aderência das distribuições Gumbel e GEV aos dados de precipitação diária

Tabela 3 - Número de indicações de melhor desempenho para as funções de densidade probabilidade, nos testes de aderência, análise de incerteza e estatísticas de ajuste.

\begin{tabular}{lccccccc}
\hline $\begin{array}{l}\text { Modelo e método de } \\
\text { ajuste }\end{array}$ & $\mathrm{KS}$ & $\chi^{2}$ & $\mathrm{Fi}$ & $\mathrm{AD}$ & $\mathrm{S}_{\mathrm{T}}$ & $\begin{array}{c}\text { Estatísticas de } \\
\text { ajuste }\end{array}$ & Total \\
\hline GEV/MM & 0 & 1 & 0 & 0 & 0 & 3 & 4 \\
GEV/ML & 4 & 1 & 0 & 3 & 3 & 3 & 14 \\
GEV/MV & 4 & 5 & 1 & 0 & 2 & 3 & 15 \\
GUM/MM & 0 & 1 & 5 & 0 & 0 & 1 & 7 \\
GUM/ML & 2 & 1 & 0 & 1 & 0 & 1 & 5 \\
GUM/MV & 0 & 2 & 5 & 3 & 6 & 0 & 16 \\
LN2/MM & 1 & 0 & 0 & 0 & 0 & 0 & 1 \\
LN2/MV & 0 & 0 & 0 & 4 & 0 & 0 & 4 \\
\hline
\end{tabular}

máxima anual no Alto do Rio Grande (MG). Os autores verificaram o melhor desempenho do método MV para as distribuições Gumbel e GEV. Alves et al. (2013) encontrou o melhor desempenho da distribuição GEV com parâmetros estimados pelo método MV em dados das precipitações máximas anuais da estação climatológica localizada em Cuiabá (MT). Esses resultados reafirmam a importância dessas distribuições probabilísticas em estudos de chuvas intensas.

Por outro lado, Franco et al. (2014) verificaram ajustes menos adequados para a distribuição Gumbel, para os três métodos de estimativa dos parâmetros (MM, ML e MV), quando comparadas com as distribuições Gama a dois parâmetros e GEV. Portanto, verifica-se a importância de se testar a aderência de distribuições de probabilidade e utilizar diferentes métodos de estimativa dos parâmetros para a escolha da melhor distribuição e verificar a magnitude do erro das estimativas de quantis de interesse através da análise de incertezas.

\section{Conclusões}

A partir dos resultados obtidos, conclui-se que: 
- O teste de aderência que mais convergiu para a indicação de fdp com melhor desempenho na análise de incerteza e nas estatísticas de ajuste foi o teste de Fi. O teste de Fi também foi o que obteve maior número de convergências quando se considera os três melhores desempenhos nas análises de incertezas e estatísticas de ajuste.

- O teste de aderência de Fi foi o mais rigoroso na seleção de distribuições de probabilidades que representem os dados, seguido pelo teste de AD, de $\chi^{2}$ e de KS.

- Os testes de KS, $\chi^{2}$ e AD indicaram a fdp com melhor desempenho na análise de incertezas em $27 \%$ das situações, enquanto que a indicação para o melhor desempenho nas estatísticas de ajuste foi observada somente para o teste de $\chi^{2}$. Quando se considera os três melhores desempenhos na análise de incerteza e nas estatísticas de ajuste, os testes de $\chi^{2}$ e $\mathrm{AD}$ apresentam vantagens em relação ao de KS.

- Apesar de mais rigoroso, o teste de Fi pode excluir fdp com bom desempenho na análise de incertezas e nas estatísticas de ajuste, o que torna os testes de $\chi^{2}$ interessante.

- As fdp com maior destaque em representar os dados de precipitações extremas na bacia do rio Sapucaí foram Gumbel com ajuste dos parâmetros realizado pelo método MV e GEV, com o ajuste dos parâmetros feito pelos métodos dos ML e MV.

\section{Referências}

ALMEIDA, L.T; ABREU, M.C.; FRAGA, M.S.; SILVA, D.D.; CECíLIO, R.A. Aspectos morfométricos relacionados ao estudo de enchentes na bacia do rio Sapucaí, Minas Gerais. Nativa, v. 5, n. 3, p.169-174, 2017.

ALVARES, C.A.; STAPE, J.L.; SENTELHAS, P.C.; GONÇALVES, J.L.M.; SPAROVEK, G. Köppen's climate classification map for Brazil. Meteorologische Zeitschrift, v. 22, n. 6, p. 711-728, 1 dez. 2013.

ALVES, A.V.P.; SANTOS, G.B.S.S.; MENEZES-FILHO, F.C. M.; SANCHES, L. Análise dos métodos de estimativa para os parâmetros das distribuições de Gumbel e GEV em eventos de precipitações máximas na cidade de CuiabáMT. Revista Eletrônica de Engenharia Civil, v. 6, n. 1, p. 32-43, 2013.

BESCOW, S.; NORTON, L.D.; MELLO, C.R. Hydrological prediction in a tropical watershed dominated by oxisols using distributed hydrological model. Water Resour Manag., v. 27, p. 341-363, 2013.

BESCOW, S.; CALDEIRA, T.L.; MELLO, C.R.; FARIA, L.C.; GUEDES, H.A. Multiparameter probability distributions for heavy rainfall modeling in extreme southern Brazil. J Hydrol., v. 4, p. 123-133, 2015.

BEN-ZVI, A. Rainfall intensity-duration-frequency relationships derived from large partial duration series. J Hydrol., v. 367 p. 104-114, 2009.

CALDEIRA, T. L.; BESKOW, S.; MELLO, C.R.; FARIA, L.C.; SOUZA, M.R.; GUEDES, H.A.S. Modelagem probabilística de eventos de precipitação extrema no estado do Rio grande do Sul. Rev. bras. eng. agríc. Ambient., v. 19, n. 3, p. 197-203, 2015.

CARVALHO, L.M.V; JONES, C.; LIEBMANN, B. The South Atlantic convergence zone: Intensity, form, persistence, and relationships with intraseasonal to interannual activity and extreme rainfall. Journal of Climate, v. 17, p. $88-108,2004$.

DAMÉ, R.C.F.; TEIXEIRA-GANDRA, C.F.A.; GUEDES, H.A. S.; SILVA, G.M.; SILVEIRA, S.C.R. Intensity-duration-frequency relationships: stochastic modeling and disaggregation of daily rainfall in the Lagoa Mirim watershed, rio grande do sul, brazil. Eng. Agríc., v. 36, n. 3, p. 492-502, 2016.

FRANCO, C.S.; MARQUES, R.F.P.V.; OLIVEIRA, A.S.; OLIVEIRA, L.F.C. Distribuição de probabilidades para precipitação máxima diária na bacia hidrográfica do rio Verde, Minas Gerais, Minas Gerais. Rev. bras. eng. agríc. Ambient., v. 18, n. 7, p. 735-735, 2014.

GARCIA, S.R.; KAYANO, M.T. Determination of the onset dates of the rainy season in central Amazon with equatorially antisymmetric outgoing longwave radiation. Theor Appl Climatol, v. 97, p. 361-372, 2009.

HOSKING J. R. M. The theory of probability weighted moments. Research Report RC 12210. Yorktown Heights (NY): IBM Research, 1986.

JUNQUEIRA-JUNIOR, J.A.; GOMES, N.M.; MELLO, C.R.; SILVA, A.M. Precipitação provável para a região de Madre de Deus, Alto Rio Grande: modelos de probabilidades e valores característicos. Ciênc. agrotec., v. 31, n. 3, p. 842-850, 2007.

JUNQUEIRA-JUNIOR, J.A.; MELLO, C.R.; ALVES, G.J. Eventos extremos de precipitação no Alto do Rio Grande, MG: Análise probabilística. Rev. bras. eng. agríc. Ambient., v. 19, n. 4, p. 301-308, 2015.

KITE, G. W. Frequency and risk analyses in hydrology. 5. ed. Highlands Ranch, Colorado: Water Resources Publications. 1988. 257p.

LOPES, T.R.; PRADO, G.; ZOLIN, C.A.; PAULINO, J.; ANTONIEL, L.S. Regionalização de vazões máximas e mínimas para a bacia do rio Ivaí - PR. Irriga, v. 21, n. 1, p. 188-201, 2016.

MARENGO, J. A.; LIEBMANN, B.; GRIMM, A. M.; MISRA, V.; SILVA DIAS, P. L.; CAVALCANTI, I. F. A.; CARVALHO, L. M. V.; BERBERY, E. H.; AMBRIZZI, T.; VERA, C. S.; SAULO, A. C.; NOGUES-PAEGLE, J.; ZIPSER, E.; SETH, A.; ALVES, L. M. Review recent developments on the South American monsoon system. International Journal of Climatology, v. 32, n. 1, p. 1-21, 2012.

MARQUES, R.F.P.V.; MELLO, C.R.; SILVA, A.M.; FRANCO, C.S.; OLIVEIRA, A.S. Performance of the probability distributions models applied to heavy rainfall daily events. Ciênc. agrotec., v. 38, n. 4, p. 335-342, 2014.

MARTINS, F.B.; PEREIRA, R.A. de A.; PINHEIRO, M.V.M.; ABREU, M.C. Desenvolvimento foliar em duas cultivares de oliveira estimado por duas categorias de modelos. Rev. Bras. Meteor., v. 29, p. 505-514, 2014. 
NAGHETTINI, M., PINTO, E.J.A. Hidrologia estatística. CPRM, Belo Horizonte, 2007, 561p

NARSKY, I. Goodness of fit: what do we really want to know? PHYSTAT 2003, p. 8-11, 2003.

PEREIRA, S.B.; NÓIA, C.P.Z.; ALMEIDA, R.A.; COELHO, C. D. Method adjustment and equation set of maximum rainfall intensity, duration and frequency in the Mato Grosso do Sul State. Eng. Agríc., v. 34, n. 4, p. 716-726, 2014.

REBOITA, M.S.; RODRIGUES, M.; SILVA, L.F.; ALVES, M.A. Aspectos climáticos do estado de Minas Gerais. Revista Brasileira de Climatologia, v. 17, p. 206-226, 2015.

REBOITA, M.S.; MARIETTO, D.M.G.; SOUZA, A.; BARBOSA, M. Caracterização atmosférica quando da ocorrência de eventos extremos de chuva na região sul de Minas
Gerais. Revista Brasileira de Climatologia, v. 21, p. 20-37, 2017.

SHIN, H.; JUNG, Y.; JEONG, C.; HEO, J.H. Assessment of modified Anderson-Darling test statistics for the generalized extreme value and generalized logistic distributions. Stoch Environ Res Risk Assess., v. 26, p. 105-114, 2011. VALVERDE, A.E.; LEITE, H.G.; SILVA, D.D.; PRUSKI, F.F. Momentos-L: teoria e aplicação em hidrologia. Rev. árvore, v. 28, n. 6, p. 927-933, 2004.

ZENG, X.; WANG, D.; WU, J. Evaluating the three methods of goodness of fit test for frequency analysis. J. Risk Anal. Crisis Response., v. 5, n. 3 p. 178-187, 2015.

This is an Open Access article distributed under the terms of the Creative Commons Attribution Non-Commercial License which permits unrestricted non-commercial use, distribution, and reproduction in any medium provided the original work is properly cited. 